\title{
Extending MCAS to hypernuclei and radiative-capture reactions
}

\author{
L. Canton ${ }^{1, a}$, K. Amos ${ }^{2}$, S. Karataglidis ${ }^{3}$, and J. P. Svenne ${ }^{4}$ \\ 1 Istituto Nazionale di Fisica Nucleare, sezione di Padova, via Marzolo 8, Padova I-35131, Italia \\ 2 School of Physics, University of Melbourne, Victoria 3010, Australia \\ 3 Department of Physics and Electronics, Rhodes University, Grahamstown 6140, South Africa \\ 4 Department of Physics and Astronomy, University of Manitoba, Winnipeg, MB, Canada R3T 2N2
}

\begin{abstract}
Using a Multi-Channel Algebraic Scattering (MCAS) approach we have analyzed the spectra of two hypernuclear systems, ${ }_{1}^{9} \mathrm{Be}$ and ${ }_{1}^{13} \mathrm{C}$. We have studied the splitting of the two odd-parity excited levels $\left(1 / 2^{-}\right.$and $\left.3 / 2^{-}\right)$at $11 \mathrm{MeV}$ excitation in ${ }_{\Lambda}^{13} \mathrm{C}$, originated by the weak $\Lambda$-nucleus spin-orbit force. We have also considered the splittings of the $3 / 2^{+}$and $5 / 2^{+}$levels in both ${ }_{\Lambda}^{9} \mathrm{Be}$ and ${ }_{\Lambda}^{13} \mathrm{C}$, finding how they originate from couplings to the collective $2^{+}$states of the core nuclei. In both hypernuclei, we suggest that there could be additional low-lying resonant states in the $\Lambda$-nucleus continua. From the MCAS approach one can extract also the full coupled-channel scattering wavefunction to be used in the calculation of various transition matrix elements. As a first application, we have considered the EM-transition matrix elements for the capture reaction $\alpha+{ }^{3} \mathrm{He} \rightarrow{ }^{7} \mathrm{Be}+\gamma$.
\end{abstract}

\section{Introduction}

In recent years, a Multi-Channel Algebraic Scattering (MCAS) approach has been developed to describe low-energy nucleonnucleus scattering, resonance phenomena, and sub-threshold spectra for medium-light nuclei. We have considered compound nuclei that are stable, or weakly bound, and have also extended the analysis to very unstable systems that are unbound with respect to proton emission, namely, that are beyond the proton drip line.

In this paper we illustrate first the possible MCAS description of the levels of two hypernuclei ${ }_{A}^{9} \mathrm{Be}$ and ${ }_{A}^{13} \mathrm{C}$. These systems are described in terms of a phenomenological $\Lambda$-light mass nucleus interaction which explicitly couples the hyperon to the collective low-lying states of the ordinary nuclear core. The phenomenological character of an appropriate $\Lambda$-light mass nucleus interaction was established in recent reviews of hypernuclear theory and experiments [1-3]. Essentially it has a central depth of 30 $\mathrm{MeV}$ (with a Woods-Saxon form) and a spin-orbit attribute considerably weaker than that of a nucleon-nucleus interaction.

The MCAS approach to particle-nucleus structure and low-energy scattering [4] emphasises the couplings of singleparticle dynamics with low-lying collective excitations of the ordinary nuclear core. The current role of these studies is to analyze bound and resonant spectra to support and interpret experimental investigations. When the Pauli principle is incorporated in nucleon-nucleus dynamics, the method has been shown to describe, consistently, the bound and resonant spectra of normal (zero-strangeness) lightmass nuclei [4-6]. Starting with the properties of spectra of non-strange nuclei, we consider the modifications to

\footnotetext{
a e-mail: luciano.canton@pd.infn.it
}

the Hamiltonians in MCAS that are required to describe hyperon-nucleus dynamics. In particular, we analyze lowlying level structures of two $p$-shell $\Lambda$ hypernuclei with regards to the structure of the hypernuclear doublet levels. We consider splittings that have been measured recently and, as well, find level structures that are just above the $\Lambda$-nucleus scattering threshold. Perhaps they may be observed in future experiments.

As a second application of the MCAS method, we consider the ${ }^{3} \mathrm{He}(\alpha, \gamma)^{7} \mathrm{Be}$ reaction cross section at astrophysical energies. The model Hamiltonian is based on a simplified 2-cluster model. The energy-dependence of the calculated astrophysical factor reproduces the experimental situation fairly well, though cross-section normalization turns out to be slightly overestimated.

\section{The two hypernuclei ${ }_{\Lambda}^{9} \mathrm{Be}$ and ${ }_{\Lambda}^{13} \mathrm{C}$}

\subsection{The $\Lambda{ }^{8} \mathrm{Be}$ interacting system}

Recent works on ${ }_{1}^{9} \mathrm{Be}$ concentrate on the ground level and on the splitting of the two first excited levels $\frac{5}{2}^{+}$and $\frac{3}{2}^{+}[1$, $3,7,8]$. These states are all of positive parity and herein we also consider those positive-parity states. Extensive theoretical work has been done in the past on low-energy negativeparity states for this system, but the relevant details are omitted herein for brevity.

To describe the $\Lambda^{-8} \mathrm{Be}$ system we applied scalings to a previously determined $n-{ }^{8} \mathrm{Be}$ Hamiltonian, adjusting the parameter values by reducing the central potential by $30 \%$, reducing the interaction radius by $15 \%$, and reducing the spin-orbit strength by an order of magnitude. In dealing with $n-{ }^{8} \mathrm{Be}$, an appropriate OPP term had been included in

This is an Open Access article distributed under the terms of the Creative Commons Attribution-Noncommercial License, which permits unrestricted use, distribution, and reproduction in any noncommercial medium, provided the original work is properly cited. 
the nucleon-core Hamiltonian [9], since the deeply bound $s$ states are already occupied by the core nucleons. However, when considering the hyperon-nucleus system, these OPP terms are removed since the hyperon is distinguishable from the nucleons and access to the deeply-bound orbits is not suppressed by Pauli exclusion.

Small adjustments were then made to give best results. The final values of the coupled-channel potential parameters are given in Table 1. Therein, we consider two sets of parameters, one with the onset of a small spin-spin component of the interaction ('Case 2'). In Table 2, we give the

Table 1. Strengths (in $\mathrm{MeV}$ ) of the $\Lambda{ }^{8} \mathrm{Be}$ interaction with $R=$ $2.3 \mathrm{fm} ., a=0.65 \mathrm{fm}$., and $\beta_{2}=0.7$

\begin{tabular}{|ccc|}
\hline \hline & Case 1 & Case 2 \\
\hline$V_{0}$ & -26.4 & -26.4 \\
$V_{\ell s}$ & 0.35 & 0.35 \\
$V_{s I}$ & 0.0 & -0.1 \\
\hline \hline
\end{tabular}

spectrum calculated with the MCAS approach and the two sets of parameters given in Table 1. We report the experimental binding energy of ${ }_{\Lambda}^{9} \mathrm{Be}$ as determined from emulsion data [10], but have also indicated in brackets the additional, newer value obtained by the E336 experiment at KEK (see Ref. [2]). While the emulsion data for ${ }_{\Lambda}^{7} \mathrm{Li}$ and ${ }_{\Lambda}^{13} \mathrm{C}$ agreed well with the KEK results, there was significant disagreement between those regarding the ${ }_{\Lambda}^{9} \mathrm{Be}$ ground-state binding energy, with the reason for this disagreement not known. Also, we consider for comparison shell-model results. These results [11] were obtained by considering not only the $\Lambda N$ and $\Sigma N$ interactions but also the $\Lambda \Sigma$ coupling. The $\Lambda$ was assumed to be in the $0 s$ orbit while the nucleons were assumed to be in the $0 p$-shell. Comparison with the bound-state spectrum obtained from MCAS is quite good.

Table 2. The positive-parity spectrum, in $\mathrm{MeV}$, of ${ }_{\Lambda}^{9} \mathrm{Be}$ (in round brackets we give the resonance widths in $\mathrm{keV}$ ). The columns labelled 'Case 1' and 'Case 2' refer, respectively, to calculations made without and with the spin-spin term in the potential. The spin-spin strength, $V_{\text {s.I }}$, was $-0.1 \mathrm{MeV}$. Comparison is also made with the results of a shell model (SM) calculation [11], where the ground state binding energy has been set to the measured value for comparison.

\begin{tabular}{|ccccc|}
\hline \hline$J^{\pi}$ & Exp. [8] & Case 1 & Case 2 & SM [11] \\
\hline$\frac{7}{2}^{+}$ & -- & $4.791(4.1)$ & $4.92(4.9)$ & \\
$\frac{9}{2}^{+}$ & -- & $4.788(4.4)$ & $4.68(3.8)$ & \\
$\frac{3}{2}^{+}$ & -3.64 & -3.70 & -3.63 & -3.66 \\
$\frac{5}{2}^{+}$ & -3.69 & -3.65 & -3.70 & -3.71 \\
$\frac{1}{2}^{+}$ & -6.71 & -6.73 & -6.73 & -6.71 \\
\hline \hline
\end{tabular}

It is interesting to observe that we get the correct size of this fine splitting between these two states with a simple phenomenological model consisting only of a central and a spin-orbit potential ('Case 1' in Table 1). Indeed, assuming no $s I$ coupling, the magnitude of the splitting between the $\frac{3}{2}^{+}$and $\frac{5}{2}^{+}$states is very small, but consistent with the separation value recently measured [12]. But the $\frac{3}{2}^{+}$state is predicted to be the lower, at variance with the recent analysis [7], where the $\frac{3}{2}^{+}$state was assessed to be the less bound of the pair.

To achieve the correct level ordering requires the introduction of a small spin-spin contribution in the $\Lambda^{-}{ }^{8} \mathrm{Be}$ phenomenological interaction ('Case 2'). We could not obtain the observed level ordering without that component in the interaction.

At $4.7 \mathrm{MeV}$ above the scattering threshold, we predict two additional positive-parity states (resonances). These are formed by coupling the $4^{+}$state in ${ }^{8} \mathrm{Be}$ with a $\Lambda$ in the $s_{\frac{1}{2}}$ state. The widths of these resonances shown in brackets in Table 2 were calculated assuming that the ${ }^{8} \mathrm{Be}$-core $4^{+}$ state has zero width. If we consider the $\alpha$-decay probability of this $4^{+}$level, then the widths reported in the table can be expected to increase quite significantly [13].

\subsection{The $\Lambda^{-12} \mathrm{C}$ interacting system}

The ${ }^{13} \mathrm{C}$ (and ${ }^{13} \mathrm{~N}$ ) systems have been studied extensively using the MCAS approach $[4,9,15,14]$. Good reproduction of the low-lying spectra, sub-threshold and resonant states, and of the elastic scattering cross-section and polarization data have been obtained with a relatively simple model Hamiltonian.

Here, we consider application of MCAS for the $\Lambda-{ }^{12} \mathrm{C}$ system. Similarly to the previous ${ }_{\Lambda}^{9} \mathrm{Be}$ case, we start with the depth of the $\Lambda$-nucleus optical potential which is is about $\frac{2}{3}$ that of the standard nucleon-nucleus one. The spinorbit strength also is much smaller, an order of magnitude smaller than that of the corresponding nucleon-nucleus system. Additionally, the potential radius is $\sim 15 \%$ smaller than used for the $n+{ }^{12} \mathrm{C}$ system. Starting from that set of parameter values, we have tuned the coupled-channel potential interaction to the description of the known spectrum. The resultant final potential parameters we have used are listed in Table 3. Again, the parameters identified as

Table 3. Strengths of the $\Lambda^{-12} \mathrm{C}$ interaction with $R=2.6 \mathrm{fm}$., $a=0.6 \mathrm{fm}$., and $\beta_{2}=-0.52$

\begin{tabular}{|ccccc|}
\hline \hline & \multicolumn{2}{c}{ Case 1 } & \multicolumn{2}{c}{ Case 2 } \\
& $\pi=-1$ & $\pi=+1$ & $\pi=-1$ & $\pi=+1$ \\
\hline$V_{0}(\mathrm{MeV})$ & -28.9 & -30.4 & -28.9 & -30.4 \\
$V_{\ell s}(\mathrm{MeV})$ & 0.35 & 0.35 & 0.35 & 0.35 \\
$V_{s I}(\mathrm{MeV})$ & 0.0 & 0.0 & -0.1 & -0.1 \\
\hline \hline
\end{tabular}

'Case 1' were those we found with the spin-spin interaction strength set to zero, while those defined as 'Case 2' had the small spin-spin interaction strength listed. The parities of the channel interactions are designated by $\pi= \pm 1$.

Four state energies have been observed and they are listed in Table 4 in the column labelled 'Exp'. A splitting 
Table 4. Spectra of ${ }_{\Lambda}^{13} \mathrm{C}$ with energies in $\mathrm{MeV}$. In round brackets we denote the corresponding widths (expressed in $\mathrm{keV}$ when using " $\mathrm{k}$ ", otherwise in $\mathrm{MeV}$ ). The shell model results are those from Ref. [16].

\begin{tabular}{|lclll|}
\hline \hline$J^{\pi}$ & Exp. [17] & Case 1 & Case 2 & SM [16] \\
\hline$\frac{1}{2}^{-}$ & --- & $+4.65(0.21)$ & $+4.66(0.23)$ & \\
$\frac{3}{2}^{-}$ & --- & $+4.64(0.22)$ & $+4.63(0.21)$ & \\
$\frac{5}{2}^{-}$ & --- & $+4.28(1.0 \mathrm{k})$ & $+4.31(1.0 \mathrm{k})$ & \\
$\frac{7}{2}^{-}$ & --- & $+4.17(1.0 \mathrm{k})$ & $+4.14(1.0 \mathrm{k})$ & \\
$\frac{3}{2}^{-}$ & --- & $+3.10(0.1 \mathrm{k})$ & $+3.15(0.1 \mathrm{k})$ & \\
$\frac{5}{2}^{-}$ & --- & $+3.05(0.1 \mathrm{k})$ & $+3.02(0.1 \mathrm{k})$ & \\
$\frac{1}{2}^{-}$ & -0.708 & -0.74 & -0.74 & \\
$\frac{3}{2}^{-}$ & -0.86 & -0.89 & -0.89 & \\
$\frac{1}{2}^{+}$ & --- & -4.12 & -4.12 & \\
$\frac{3}{2}^{+}$ & -6.81 & -7.177 & -7.08 & -6.22 \\
$\frac{5}{2}^{+}$ & -6.81 & -7.178 & -7.24 & -6.19 \\
$\frac{1}{2}^{+}$ & -11.69 & -11.68 & -11.68 & -10.95 \\
\hline
\end{tabular}

between the $\frac{3}{2}^{+}$and $\frac{5}{2}^{+}$states is not resolved as yet. The theoretical spectra, however, contain a richer structure as shown by the results listed under the 'Case 1' and 'Case 2 ' columns in the table. Both model calculations predict a $\frac{1}{2}^{+}$bound state at $4.12 \mathrm{MeV}$ below threshold. As the spin-spin interaction has no effect upon its excitation energy, this state corresponds to an $s_{\frac{1}{2}}-\Lambda$ coupled to the $0_{2}^{+}$ state at $7.65 \mathrm{MeV}$ in ${ }^{12} \mathrm{C}$, which is an highly exotic state as it corresponds to the coupling of the hyperon to the superdeformed Hoyle state. This state is not predicted by the shell model [16], as the $0 p$-shell model of the underlying structure of ${ }^{12} \mathrm{C}$ cannot predict the Hoyle state. The ground state and low-lying spectrum from the shell model for ${ }_{\Lambda}^{13} \mathrm{C}$, however, agree generally well with the predictions from MCAS. The $\frac{1}{2}^{+}$state we expect at $7.56 \mathrm{MeV}$ excitation in ${ }_{\Lambda}^{13} \mathrm{C}$ has been placed much higher in excitation (12.2 $\mathrm{MeV}$ ) in the cluster model evaluations [18]. That is due to the strong state dependence of the $\Lambda$-nucleus interaction, found by $s$-wave folding in that model [19]. We have still to wait for more detailed experimental investigations of that spectrum to decide whether it is a strong state dependence effect or simply the extremely weak natural excitation of the second $0^{+}$from the ground state of ${ }^{12} \mathrm{C}$ that explains the position of a second $\frac{1}{2}^{+}$state in the spectrum of this hypernucleus. Note that a similar state has been predicted by MCAS calculations of $n+{ }^{12} \mathrm{C}$ to be in the spectrum of the non-strange ${ }^{13} \mathrm{C}$. That state has spin-parity $J^{\pi}=\frac{1}{2}^{-}$, lies $2.68 \mathrm{MeV}$ above the scattering threshold, and though unobserved, is partner to a mirror state of such structure in ${ }^{13} \mathrm{~N}$ at $6.97 \mathrm{MeV}$ above the $p-{ }^{12} \mathrm{C}$ threshold. That is 1.3 $\mathrm{MeV}$ higher than where a $J^{\pi}=\frac{1}{2}^{-}$state has been observed in ${ }^{13} \mathrm{~N}$.

Additionally, a set of six odd-parity states of ${ }_{A}^{13} \mathrm{C}$ are predicted to be just a few MeV above the scattering threshold. They are states formed by coupling of a $p_{\frac{3}{2}}$ - and of a $p_{\frac{1}{2}}-\Lambda$ with the $2_{1}^{+}$state in ${ }^{12} \mathrm{C}$. Without deformation (and no spin-spin interaction) they would form a degenerate quar- tet $\left(\frac{1}{2}^{-}, \frac{3}{2}^{-}, \frac{5}{2}^{-}\right.$, and $\left.\frac{7}{2}^{-}\right)$and a degenerate doublet $\left(\frac{3}{2}^{-}\right.$and $\left.\frac{5}{2}^{-}\right)$of states. Deformation and spin-spin effects then break those degeneracies and mix states of like spin-parity. Since these six states are embedded in the $\Lambda-{ }^{12} \mathrm{C}$ continuum, they are resonances and their widths are listed (in brackets) in Table 4 . These resonance states are very narrow save for the doublet of $\left.\frac{3}{2}^{-}\right|_{3}$ and $\left.\frac{1}{2}^{-}\right|_{2}$ resonances. We have also calculated the corresponding excitation function, assuming a low-energy $\Lambda-{ }^{12} \mathrm{C}$ elastic scattering process.

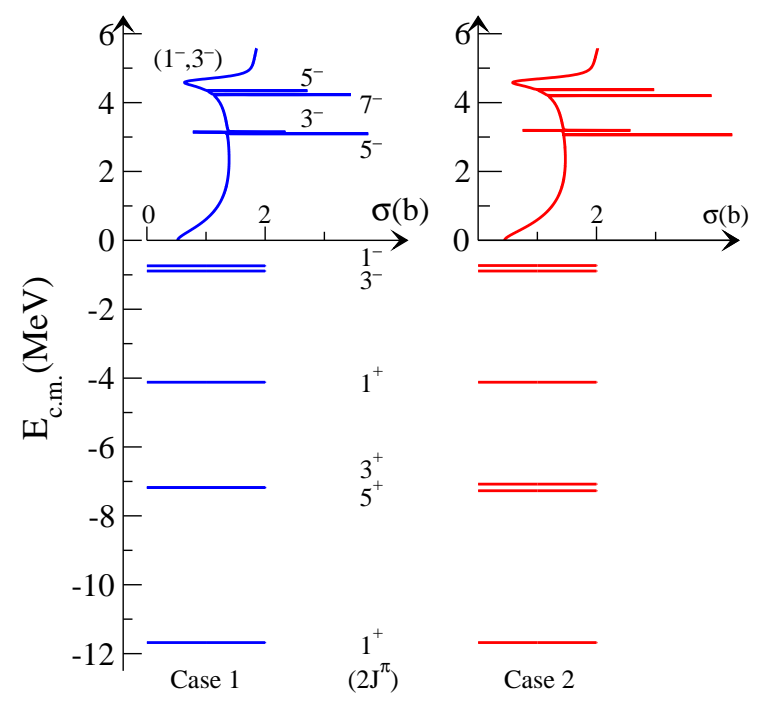

Fig. 1. The spectra and total elastic cross sections for $\Lambda^{-12} \mathrm{C}$ scattering resulting from MCAS calculations made using two sets of parameter values.

The spectra for ${ }_{\Lambda}^{13} \mathrm{C}$ is given in Fig. 1 . Below the $\Lambda-{ }^{12} \mathrm{C}$ threshold the discrete bound states are shown while above that threshold the theoretical cross sections are given for both the case of $V_{s I}=0.0$ and $-0.1 \mathrm{MeV}$. Of prime interest is the fine splitting of the bound $\frac{1}{2}^{-}$and $\frac{3}{2}^{-}$levels, observed respectively at $10.98 \mathrm{MeV}$ and $10.83 \mathrm{MeV}$ above the ground state [12]. We note that there is also a very small splitting expected between two bound levels, $\frac{5}{2}^{+}$and $\frac{3}{2}^{+}$, at the excitation energy of $\sim 4.88 \mathrm{MeV}$. Our exploratory calculation suggests a splitting of $\sim 160 \mathrm{keV}$ for both doublets. Experimentally, the splitting of the $\frac{1}{2}^{-}$and $\frac{3}{2}^{-}$states was found to be $152 \pm 54 \pm 36 \mathrm{keV}$. The splitting of the more bound positive-parity states has not been determined quantitatively to date.

One can extract the $\Lambda$-nucleus spin-orbit strength from the splitting of the $\frac{1}{2}^{-}$and $\frac{3}{2}^{-}$states. Using the experimental information of a $152 \mathrm{keV}$ splitting for the two oddparity levels, we settle upon a $\Lambda$-nucleus spin-orbit strength of $0.35 \mathrm{MeV}$. We also conclude that the current knowledge of experimental spectra is insufficient to assess any importance of the $\Lambda$-nucleus $V_{s I}$ coupling in ${ }_{\Lambda}^{13} \mathrm{C}$. For this reason, for the $\frac{5}{2}^{+}, \frac{3}{2}^{+}$level splitting we considered the ${ }_{\Lambda}^{9}$ Be system for which accurate experimental information on the splitting of such levels has been obtained [2]. That information 
leads to a significant constraint on the $\Lambda$-nucleus $V_{s I}$ coupling.

\section{Test of radiative-capture calculation with MCAS}

In evaluation of radiative-capture reactions, we have to determine the (matrix-like) scattering wave function for bound and continuum states. In the case of the initial continuum state, this can be rewritten in closed form in terms of regu$\operatorname{lar}\left(F_{L}\right)$, irregular $\left(G_{L}\right)$, and outgoing $\left(O_{L}^{(+)}\right)$Coulomb functions, and a matrix-inversion operation. This matrix inversion in the wavefunction expression retains all the resonant and nonresonant nuclear structures relevant for lowenergy capture and remove the need of formalisms (like Rmatrix) which merely parametrize known resonances. The electro-magnetic (EM) operator of single-photon emission is constructed via a generalization of the Siegert theorem, which provides the amplitude of the electromagnetic process in an explicitly gauge-independent way. A multipole decomposition of the relevant Electric and Magnetic transition matrix elements and the corresponding results are given in Refs. [20,21]. Theoretical results are compared in Fig. 2 with recent LUNA data as well as with other data sets (full list is given in Ref. [20]). Our results reproduce satisfactorily the energy dependence but normalization appears overestimated $(\simeq 1.4)$, and this represents a typical aspect of two-cluster model calculations.

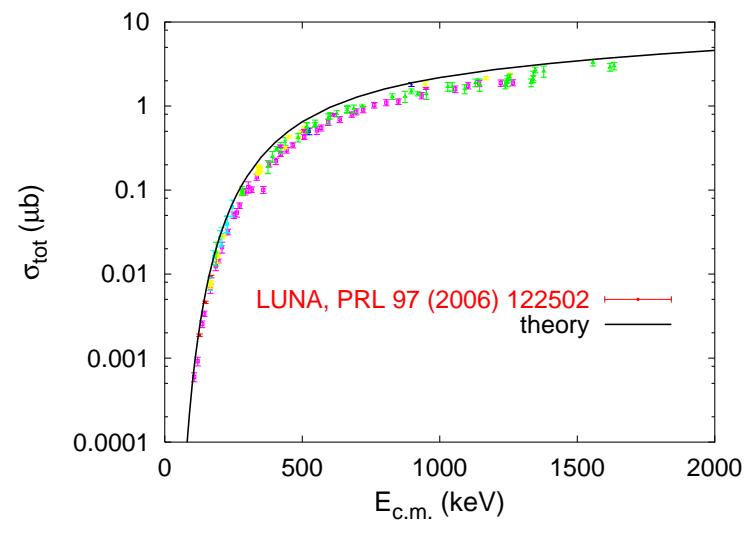

Fig. 2. Total cross section of ${ }^{3} \mathrm{He}(\alpha, \gamma)^{7} \mathrm{Be}$ reaction.

\section{Summary and Conclusions}

We have applied the MCAS approach to study the excitation spectra of light hypernuclei ${ }_{\Lambda}^{9} \mathrm{Be}$ and ${ }_{\Lambda}^{13} \mathrm{C}$. The theoretical approach emphasises the single-particle motion of the hyperon in the mean-field of the ordinary nuclear core, which is however coupled to its own low-lying collective motions.
The phenomenological hyperon-core potential was constructed from the ordinary nucleon-core potential by removing Pauli-blocking effects, making a $15 \%$ shrinkage of the radius, using a $\frac{2}{3}$ reduction of the strength of the central potential, and reducing drastically the spin-orbit potential. However, the coupling of the single hyperon with the collective motion of the core is rather important in defining the hypernuclear spectra. The deformation parameter is essentially that required in the associated nucleon-nucleus dynamics.

From the recently observed fine splitting of the excited hypernuclear spectra for these two nuclei, we conclude that the $\frac{1}{2}^{-}-\frac{3}{2}^{-}$splitting in ${ }_{\Lambda}^{13} \mathrm{C}$ is dominated by the $\Lambda$-core spinorbit interaction. On the other hand, the fine splittings of the $\frac{3}{2}^{+}-\frac{5}{2}^{+}$doublet in both ${ }_{\Lambda}^{9} \mathrm{Be}$ and ${ }_{\Lambda}^{13} \mathrm{C}$ are generated by the coupling of the $s_{\frac{1}{2}}-\Lambda$ single-particle motion with the $2^{+}$ collective excitation of the core. As it involves an $s$-wave $\Lambda$-particle, it is independent of the spin-orbit interaction. The recently measured structure of the fine $\left(\frac{3}{2}^{+}-\frac{5}{2}^{+}\right)$splitting in ${ }_{1}^{9} \mathrm{Be}$ enabled us to determine the sign and strength of that spin-spin interaction, $V_{s I}$.

We have also outlined the theoretical scheme to apply the MCAS approach to radiative capture, following Refs. [20,21]. We have considered in particular the radiativecapture reaction $\alpha+{ }^{3} \mathrm{He} \rightarrow{ }^{7} \mathrm{Be}+\gamma$, which can be reasonably described with a two-cluster model. Our model calculation tests the feasibility of radiative-capture calculation within the Hamiltonian-type MCAS scheme and the construction of EM operators that are explicitly gauge independent.

\section{References}

1. D. J. Millener, Lect. Notes Phys. 724, 31 (2007).

2. O. Hashimoto and H. Tamura, Prog. Part. Nucl. Phys. 57, 564 (2006).

3. H. Tamura et al., Nucl. Phys. A804, 73 (2008).

4. K. Amos et al., Nucl. Phys. A728, 65 (2003).

5. L. Canton et al., Phys. Rev. Lett. 96, 072502 (2006).

6. L. Canton et al., Phys. Rev. C 74, 064605 (2006).

7. H. Tamura, Nucl. Phys. A752, 155c (2005).

8. H. Akikawa et al., Phys. Rev. Lett. 88, 082501 (2002).

9. L. Canton et al., Phys. Rev. Lett. 94, 122503 (2005).

10. M. K. Juric et al., Nucl. Phys. B52, 1 (1973).

11. D. J. Millener, Nucl. Phys. A754, 48c (2005).

12. H. Tamura et al., Nucl. Phys. A754, 58c (2005).

13. P. Fraser et al., Phys. Rev. Lett. 101, 0242501 (2008).

14. J. P. Svenne et al., Phys. Rev. C 73, 027601 (2006).

15. L. Canton et al., Nucl. Phys. A790, 251c (2007).

16. D. J. Millener, private communication (2009).

17. H. Kohri et al., Phys. Rev. C 65, 034607 (2002).

18. E. Hiyama et al., Phys. Rev. Lett. 85, 270 (2000).

19. Y. Yamada et al., Prog. Theo. Phys. Japan (supp) 81, 104 (1985).

20. L. Canton and L.G. Levchuk, Nucl. Phys. A808, 192 (2008).

21. L. Canton, L. Levchuk, and A.V. Shebeko, Few Body Syst. 44, 357 (2008). 